\title{
Kurse im zweiten Halbjahr 2019
}

\begin{tabular}{|c|c|c|}
\hline $\begin{array}{l}\text { Do 12.-So } 15.9 . \\
\text { (Kurs 243) }\end{array}$ & $\begin{array}{l}\text { Refresher-Kurs Bewegungsapparat RKBWA-02-2019 } \\
\text { Thema: obere Extremität, SGUM-anerkannt }\end{array}$ & Dr. Beat Dubs \\
\hline $\begin{array}{l}\text { So 22.-Sa } 28.9 . \\
\text { (Kurs 257) }\end{array}$ & $\begin{array}{l}\text { Intensiv-Wochen-Trainingskurs Bewegungsapparat } \\
\text { WKBWA-09-2019 }\end{array}$ & Dr. Beat Dubs \\
\hline $\begin{array}{l}\text { Do 10.-So } 13.10 . \\
\text { (Kurs 258) }\end{array}$ & $\begin{array}{l}\text { Aufbaukurs Sonografie des Bewegungsapparats } \\
\text { AKBWA-02-2019 }\end{array}$ & Dr. Beat Dubs \\
\hline $\begin{array}{l}\text { Do 24.-So } 27.10 . \\
\text { (Kurs 259) }\end{array}$ & $\begin{array}{l}\text { Intensiv-Trainingskurs Bewegungsapparat } \\
\text { TKBWA-10-2019 }\end{array}$ & Dr. Beat Dubs \\
\hline $\begin{array}{l}\text { Do } 31.10 .- \text { So } 3.11 . \\
\text { (Kurs 260) }\end{array}$ & $\begin{array}{l}\text { Intensiv-Trainingskurs Abdomen und Weichteile } \\
\text { TKABDA-11-2019 }\end{array}$ & Dr. Beat Dubs \\
\hline $\begin{array}{l}\text { So } 24.11 .- \text { Sa } 30.11 . \\
\text { (Kurs 261) }\end{array}$ & $\begin{array}{l}\text { Intensiv-Wochen-Trainingskurs Bewegungsapparat } \\
\text { WKBWA-12-2019 }\end{array}$ & Dr. Beat Dubs \\
\hline $\begin{array}{l}\text { Do 12.-So } 15.12 . \\
\text { (Kurs 262) }\end{array}$ & $\begin{array}{l}\text { Intensiv-Trainingskurs Bewegungsapparat } \\
\text { TKBWA-13-2019 }\end{array}$ & Dr. Beat Dubs \\
\hline \multicolumn{3}{|c|}{ Kurse im ersten Quartal 2020} \\
\hline $\begin{array}{l}\text { Do 9.-So } 12.1 . \\
\text { (Kurs 264) }\end{array}$ & $\begin{array}{l}\text { Intensiv-Trainingskurs Bewegungsapparat } \\
\text { TKBWA-01-2020 }\end{array}$ & Dr. Beat Dubs \\
\hline $\begin{array}{l}\text { So } 26.1 .- \text { Sa } 1.2 . \\
\text { (Kurs 265) }\end{array}$ & $\begin{array}{l}\text { Intensiv-Wochen-Trainingskurs Bewegungsapparat } \\
\text { WKBWA-02-2020 }\end{array}$ & Dr. Beat Dubs \\
\hline $\begin{array}{l}\text { Do 6.-So 9.2. } \\
\text { (Kurs 266) }\end{array}$ & $\begin{array}{l}\text { Sonographie en médecine du sport RKSPM-01-2020 } \\
\text { (deutsch und französisch) }\end{array}$ & $\begin{array}{l}\text { Dr. Catherine Heim und } \\
\text { Dr. Beat Dubs }\end{array}$ \\
\hline $\begin{array}{l}\text { Do 13.-So } 16.2 \text {. } \\
\text { (Kurs 267) }\end{array}$ & $\begin{array}{l}\text { Grund- und Aufbaukurs Säuglingshüfte nach Graf } \\
\text { GKSGH-01-2020 }\end{array}$ & Dr. Beat Dubs \\
\hline $\begin{array}{l}\text { Do 27.2.-So } 1.3 . \\
\text { (Kurs 268) }\end{array}$ & $\begin{array}{l}\text { Grundkurs Sonografie des Bewegungsapparats } \\
\text { GKBWA-01-2020 (deutsch und englisch) }\end{array}$ & Dr. Beat Dubs \\
\hline $\begin{array}{l}\text { So } 1 .- \text { Sa } 7.3 . \\
\text { (Kurs 269) }\end{array}$ & $\begin{array}{l}\text { Intensiv-Wochen-Trainingskurs Bewegungsapparat } \\
\text { WKBWA-03-2020 }\end{array}$ & Dr. Beat Dubs \\
\hline
\end{tabular}

Weitere Kurse sind noch geplant, Einzelheiten folgen auf der Webseite.

Das gesamte Angebot und die Einzelheiten zu den Kursen sind auch unter www.ungarnkurse.ch im Internet aufgeschaltet. 